\title{
Pediatric Care for HIV
}

\author{
Sumathi $\mathrm{R}^{1^{*}}$, Gladys Kalpana ${ }^{2}$, Junaid Rahman $\mathrm{P}^{3}$, Ramya. $\mathrm{S}^{4}$ \\ ${ }^{1}$ Associate Professor, Department of Pharmaceutical Biotechnology, Nandha College of \\ Pharmacy, Erode, Tamilnadu, Email:sumoraji@gmail.com \\ ${ }^{2}$ Associate Professor, Department of Pharmacology, Nandha College of Pharmacy, \\ Erode, Tamilnadu, Email: gladyskalpana@nandhapharmacy.org \\ ${ }^{3}$ Assistant Professor, Department of Pharmacology, Nandha College of Pharmacy, \\ Erode, Tamilnadu, Email: junaidrahman@nandhapharmacy.org \\ ${ }^{4}$ Associate Professor, Department of Medical Surgical Nursing, Nandha College of \\ Nursing, Erode, Tamilnadu, Email:Ramyasampath2345@gmail.com \\ ${ }^{*}$ Corresponding Author
}

\begin{abstract}
The growing number of pediatric AIDS diagnoses need immediate action to ensure the children's life and well-being. The purpose of this paper is to advocate aimed at the treatment of HIV-infected toddlers and kids by utilizing the availability of antiretroviral therapy (ART). Final ART can boost the period and provide a high level of satisfaction in their life. Antiretroviral (ARV) therapy had better be delivered as measure of a broad package of pediatric HIV care. Pediatricians must afford mundane precaution and work diligently with pediatric HIV consultants to get insight into HIV development and, as a result, the basic for ART.
\end{abstract}

Keywords: Pediatric, HIV, Care

\section{Introduction}

Medication assays are indicated for examining plasma HIV polymer plasma HIV ribonucleic acid plus resistant intricate disassociated p24 antigen in kids under the age of eighteen months. Medicine examinations devour in recent times grown to be precise simpler, a reduced amount of affluent, and further trustworthy. Infants with a positive medical specialty check at 48 hours of age may have an intrauterine infection. Infants that get a deleterious virological assessment for the period of the leading week of life time but later have a positive test have an intrapartum septicity. HIV infection is identified by HIV DNA or polymer detection in the majority of infected nonbreast feeding newborns by one month of age and in virtually all infected babies by six months of age. Because of the risk of contamination from maternal blood, blood samples from the membrane should not be used for diagnostic purposes. If alternative feeding isn't available and a child is breastfeeding, medication tests are performed at any time. If the result is negative, the test must be repeated six weeks after the newborn's complete interruption of breastfeeding to ensure that the infant is not HIV-infected. Most opposedseptic HIV-exposed children can lose mother opposed bodies by the age of twelve months. A positive result for HIV supermolecule testing in a child of this age usually indicates HIV infection. Decisive HIV verdict in children 18 months old (whether HIV exposure is suspected or known) is usually accomplished using macromolecule tests (ELISA or rapid test), whereas Western Blot has been utilised in the bygone, corroboration of HIV eminence is further steadfastly proven with medication testing.

\section{Evaluation of HIV infested children's}

Entirely kids and children who have been identified with HIV infection should have quantifiable and work shop tests on the road to manage the stage of HIV clinical disease and immunodeficiency, eligibility for ART, and any morbidities or concerns that need to be addressed. This baseline examination will provide an opportunity to begin cotrimoxazole anticipatory medical aid and can fill a niche in providing message and upkeep to sick kids in addition their parentages/caregivers. Clinical and laboratory evaluation of children with HIV should include laboratory tests such as complete blood count, helper T lymphocyte count, bilirubin, creatinine, urinalysis, and glucose testing for tuberculosis, viral hepatitis, and $\mathrm{C}$ (if at risk), and maternity checks for pubertal girls. Organic process assistance had to consist of early measures to ensure enough nutrient aperture, supported in the neighborhood presented and lowcost foods, and also the delivery of micronutrients comparable to the required daily amount. A supplement should be administered in accordance with the WHO-recommended high-dose dosing schedule for children at high risk2 of deficiency. Clinical findings revealed that newborns with AIDS developing clinical disease frequently have a transitory genetic abnormality as well as cow's milk molecule (CMP) intolerance. Consultants typically 
recommend that if a kid has severe diarrhoea, special milk formulae and lactose CMP free milk, if available, can help to alleviate the condition. Before initiating HIV-infected children on ART, parents and/or other caregivers must be counselled on a variety of issues. Adherence to ART is the key to success in treatment. It predicts and modifies medication as well as clinical response to treatment, and its significance should be communicated to caregivers. The goals of such a communication should include creating trust with the caregiver and establishing mutually agreed care goals. Correct nutrition, as well as the optimum use of local foods, appropriate nutrition supplements, and the organic process management of HIV-related illnesses impacting appetite and ability to eat, is a primary counselling concern. When a child hits puberty, a number of topics must be addressed throughout the message to ensure that appropriate treatment and care is being provided. They are currently maturing physically, psychologically, and sexually, which has ramifications for their treatment and care.

\section{ART in children's}

The most significant dispute in clinically running HIV infected children is starting long-term antiretroviral therapy (ART). The therapeutic drug's effectiveness in lowering HIV-related indisposition and death in newborns and children is comparable to that discovered in adults. However, there are special issues for HIV-infected newborns and children who have been exposed to ZDV and NVP, as well as other ARVs used during pregnancy, which may result in ARV resistance and age reliant variations in medication markers (e.g. CD4 share is used for children, not CD4 count). ART should be started when a child has an AIDS-defining pathological condition or severe medication failure. The choice to start ART must be decided based on individual CD4 share and age. Based on these criteria, the situation is at this moment potential to compute the precise chance of progress to AIDS or death over a period of years. Infants at high risk of clinical progression, especially HIV encephalopathy, should begin ART with a greater CD4 share than mature children. To aid in the introduction of ART in children with a confirmed HIV diagnosis, the WHO recommendations for clinical staging of medical HIV/AIDS should be employed. HIV progression in youngsters may be much faster than in adults. Because the predictive significance of individual HIV compound levels for disease progression is difficult to evaluate, particularly in newborns, an assessment of being load (VL) isn't required before starting treatment. VL, on the other hand, remains a good activity of treatment response and will be conducted prior to starting ART as well as one month and three months of cure, if practicable. The goal of treatment is to achieve an indiscernible VL level, which prevents infective agent reproduction and lowers the likelihood of resistance to the ART combination utilised. The criteria for selecting first-line ARV procedures for infants and children are the equivalent as for adults, with a few extra factors such as the patient's age, the quality of pharmaceutical originations, and the side-effect summary being taken into account.

\section{ARV drug dosage and monitoring}

The ARV medicine dose must be evaluated and modified every three months in accordance with the kid's encumbrance or else, around be situated a threat of underdoing and rising conflict. Prescriptions are considered as a weight unit per metric weight unit weight or as a metric weight unit per square metre body surface. Standardization is critical. Children with HIV should be observed on a regular basis in demand to regulate circumstance managing strategies and action programs. Such observance should include both individuals who are not eligible for ART and those who are receiving treatment. Clinical evaluations of babies and children who are not yet eligible for ART should be performed every 3-6 months. Advance catastrophe is one of the most prevalent AIDS significant symptoms in medical science HIV, thus all children should be planned on an advance graph. Every encounter with children and caregivers, ideally once a month, should include analysis and organic process support.

Table 1: Threshold level for ART treatment initiation

\begin{tabular}{|l|l|l|l|l|}
\hline $\begin{array}{l}\text { Immunological } \\
\text { marker (CD4 } \\
\text { count) }\end{array}$ & $\leq 11$ months & $12-35$ months & $36-59$ months & $\geq 5$ years \\
\hline & $\begin{array}{l}\leq 25 \%(\leq 1500 \\
\text { cells } / \mathrm{mm} 3)\end{array}$ & $\begin{array}{l}\leq 20 \%(\leq 750 \\
\text { cells } / \mathrm{mm} 3)\end{array}$ & $\begin{array}{l}\leq 15 \%(\leq 350 \\
\text { cells } / \mathrm{mm} 3)\end{array}$ & $\begin{array}{l}\leq 200 \mathrm{cells} / \mathrm{mm} 3 \\
(\leq 15 \%)\end{array}$ \\
\hline
\end{tabular}

In comparison to younger children, adherence may improve the miniscule kids are 8-10 years old at the time of identification. The children's age, maturity, and social conditions must be considered, and communiqué had better take place in a phonological and grade level that they apprehend. Youngsters' upkeep groups, in which children arise to the clinic and play activities together, could potentially be beneficial. They discover they are not the only ones living with HIV, and their carers have the prospect to communicate with one another. Systematic examination of process standing and associated symptoms is critical to early disease detection and poor growth, and it should be part of routine clinical monitoring of HIV-infected infants and children. 
Process analysis should be performed monthly on newborns and every three months on different children, and should include: manner of feeding, frequency, amount or quantity taken, adequacy of supply, gut and pee habits, and reported difficulties. It is crucial to test and continue to monitor the development of infants' and young children's cognitive, motor, language, and social skills, as a large number of them exhibit early and marked deficits in these areas, which may be critical early indications of HIV ill health progression. . The primary goal is to uncover organic process flaws early on, allowing for intervention to prevent or lessen the impact of severe problems. The optimal care of HIVinfected children necessitates the use of just ART. Children with extremely low CD4 levels for their age are the most vulnerable to Ols. When allowing for preventive treatment in a child who has recently presented and is extremely agitated, the primary objective is to begin in effect ART to refurbish the immune response. Acceptable $\mathrm{Ol}$ hindrance is extremely critical for adolescents who have failed many ART regimens and have very low CD4 counts, regardless of whether or else not they are at this time on ART.

\section{Conclusion}

Ache in children by means of HIV AIDS may be a multidimensional, biologically intricate disadvantage that leads to a lower eminence of life and an increase in impermanence. Pain management, pain treatment, and the palliative use of anesthetics and downers are all critical phases of precaution for an HIV-infected child. Regardless of advances in HIV treatment and care in children, pain is expected to complicate medical controlling and make smaller quality of life for a proportion of children with advanced disease. Because pain in this population is often complex, optimal care will require the coordinated participation of several professionals, as well as anesthesiologists, pain consultants, social personnel, nursing workforces, and others.

\section{References}

1. Moodley $\mathrm{D}$ et al. Predicting perinatal human immunodeficiency virus infection by antibody patterns. The Pediatric Infectious Disease Journal, 1995, 14(10):850-852.

2. Management of a child with a serious infection or malnutrition: guidelines for the care at the firstreferral level in developing countries. Geneva, World Health Organization, 2000.

3. Management of serious malnutrition: a manual for physicians and other senior health workers. Geneva, World Health Organization, 1998.
4. Nutrient requirements for people living with HIV: report of a technical consultation. Geneva, World Health Organization, 2003.

5. Miller TL. Nutritional aspects of HIV-infected children receiving highly active antiretroviral therapy. AIDS, 2003, 17(Suppl. 1):S130-S140.

6. Vitamin A supplements: a guide to their use in the treatment and prevention of vitamin A deficiency and xerophthalmia, 2nd ed. Geneva, World Health Organization, 1997.

7. Coutsoudis A et al. The effects of vitamin A supplementation on the morbidity of children born to HIV infected women. American Journal of Public Health, 1995, 85(8):1076-1081.

8. Sfawzi WW et al. A randomized trial of vitamin A supplements in relation to mortality among human immunodeficiency virus-infected and uninfected children in Tanzania. The Pediatric Infectious Disease Journal, 1999, 18(2):127-133. 432 HIV/AIDS TREATMENT AND CARE CLINICAL PROTOCOLS FOR THE WHO EUROPEAN REGION

9. Van Dyke RB et al. Reported adherence as a determinant of response to HAART in children who have HIV-infection. Pediatrics, 2002, 109:e61.

10. De Martino $M$ et al. Reduction in mortality with availability of antiretroviral therapy for children with perinatal HIV-1 infection. Italian Register for HIV Infection in Children and the Italian National AIDS Registry. JAMA, 2000, 284:190-197.

11. Phase IIB trial to evaluate the efficacy of oral nevirpaine and the efficacy of oral AZT in infants born to HIV-infected mothers in Uganda for prevention of vertical HIV transmission (Version 2.0). (HIVNET 012) HIVNET/HPTN Group, 14 May 2003, Seattle, Washington, USA (http://www.hptn. org/Web\%20Documents/HIVNET_Protocols/HIVNET_01 2.pdf

12. Short-term risk of disease progression in HIV-1-infected children receiving to antiretroviral therapy or zidovudine monotherapy: a meta-analysis. HIV Paediatric Prognostic Markers Collaborative Study Lancet 2003; 362:1605-11.

13. Use of total lymphocyte count for informing when to start antiretroviral therapy in HIV-infected children: a meta-analysis of longitudinal data. HIV Paediatric Prognostic Markers Collaborative Study. Lancet 2005; 366:1868-74.

14. HIV Paediatric Prognostic Markers Collaborative Study [web site]. London, Medical Research Council Clinical Trials Unit, 2006 (http: //www.hppmcs.org, accessed 8 June 2006).

15. Sharland $M$ et al. PENTA guidelines for the use of antiretroviral therapy. HIV Medicine, British HIVAssociation, 5(S2):61-86, 2004. 